\title{
Assessing uncertainty in outsourcing clinical services at tertiary health centers
}

\author{
John E. Billi ${ }^{1}{ }^{*} \dagger$, Chih-Wen Pai ${ }^{2, \ddagger}$ and David A. Spahlinger ${ }^{1, \S}$ \\ ${ }^{1}$ University of Michigan Medical School, Michigan, USA \\ ${ }^{2}$ University of Michigan Health Management Research Center, Michigan, USA
}

\section{SUMMARY}

When tertiary health centers face capacity constraint, one feasible strategy to meet service demand is outsourcing clinical services to qualified community providers. Clinical outsourcing enables tertiary health centers to meet the expectations of service timeliness and provides good opportunities to collaborate with other health care providers. However, outsourcing may result in dependence and loss of control for the tertiary health centers. Other parties involved in clinical outsourcing such as local partners, patients, and payers may also encounter potential risks as well as enjoy benefits in an outsourcing arrangement. Recommendations on selecting potential outsourcing partners are given to minimize the risks associated with an outsourcing contract. Copyright (C) 2006 John Wiley \& Sons, Ltd.

KEY WORDS: organizational change; decision making; strategic planning; tertiary health center; outsourcing

\section{INTRODUCTION}

Introduction of the Medicare Perspective Payment System, adoption of managed health care, and payment reduction in public programs in the United States have contributed to hospital closures, mergers, and downsizing in 1990s, creating an impression of hospital capacity constraint. Despite pitfalls in calculating occupancy rates (Green, 2002) and a federal target of 85\% occupancy, a general agreement among hospital administrators is that operational inefficiency occurs if occupancy rates exceed over $80 \%$. An analysis from the Center for Studying Health System Change's (HSC) Community Tracking Study (CTS) reports average inpatient occupancy rates below $70 \%$ from 1985 to 2001 among community hospitals in the

\footnotetext{
* Correspondence to: Dr J. E. Billi, M7319 Medical Science Building I, 1301 Catherine, Ann Arbor, MI 48109-0624, USA. E-mail: jbilli@umich.edu

${ }^{\dagger}$ Professor of Internal Medicine and Medical Education, Associate Dean for Clinical Affairs, Associate Vice President for Medical Affairs.

${ }^{\ddagger}$ Senior Research Associate Office of the Dean.

${ }^{\S}$ Clinical Associate Professor of Internal Medicine, Executive Medical Director of Faculty Group Practice, Senior Associate Dean for Clinical Affairs.
} 
United States (Bazzoli et al., 2003). Considering other factors, this CTS report suggests a maldistribution of inpatient capacity in some markets, instead of a general lack of hospital beds.

Besides mismatch between supply and demand for hospital beds, perceived capacity constraint is also manifested in long waits for physician appointments. Institute of Medicine in Cross the Quality Chasm identifies timeliness of care as one of the six dimensions for improvement in health care (Institute of Medicine, 2001). Findings from HSC Household Survey report an upward trend of waiting time for physician appointments between 1997 and 2001 (Strunk and Cunningham, 2001). Results from the 2001 Medical Expenditure Panel Survey (MEPS) indicate that $44.5 \%$ of adults with private insurance coverage in the United States were unable to receive care as soon as they wanted when needing urgent care (Greenblatt, 2002).

Tertiary health centers often provide a wide scope of clinical services while maintaining their missions of education and research. When demands for clinical services exceed capacity to provide timely care, access becomes a concern and patients must choose between long waits or seeking care from less familiar providers. When facing capacity constraint, often measured as high occupancy rates and long waits for appointments, a tertiary health center has options of turning down new demand, expanding its capacity to meet all the demand, or establishing a contractual relationship (or outsourcing) with local competing institutions for excess demand. How should a tertiary health center make strategic decisions?

Pushing away patients or sustaining long delays for services may be proved detrimental both to public relation and quality of patient care. Expanding capacity requires investment in workforce, infrastructure, and space. Substantial investment can be prohibitively expensive, time consuming, and therefore not practical to solve the urgent capacity issue. As a result, strategic outsourcing may provide a feasible solution without costly expansion while still preserving a tertiary health center's commitment to its patient population, especially when there is excess capacity in the community.

In a multi-payer health care system reserving capacity through outsourcing is especially financially critical to capacity constrained health care providers. In the United States, about $64 \%$ of the population obtained private health insurance through their employers in 2004, while 19\% obtained it through public programs (Medicare and Medicaid), with the remaining 17\% uninsured (Center for Financing, Access, and Cost Trends, 2004). Historically, private fee-for-service insurance plans tend to have more lucrative payment rates than public programs or private managed care plans. If a provider has limited capacity and, therefore, long waits, individuals with private fee-for-service insurance coverage can afford to seek care from clinicians and facilities that have capacity. Since patients in lower paying managed care and government programs have fewer choices of providers, they are more likely to just tolerate the waits. Ultimately, such provider shopping by private fee-for-service consumers collectively results in worsening payer mix and financial stress for any health care providers who have limited available capacity. Thus, it is vital for health care providers to pursue strategies, such as clinical outsourcing, in order to maintain financial health while preserving its commitment to community services.

In addition to financial relevance to providers, outsourcing also is important from a policy perspective. In many industrialized countries, at least at present, health care 
facilities are either government owned or funded. In the United States the majority of health care facilities are privately owned, though many are not for profit. Even so, a few states have regulations in place for the purpose of regional planning and control of health care resources. One such regulation in some states is the Certificate of Need $(\mathrm{CON})$, which requires health care providers to obtain regulatory permission for capital investment for construction of new facilities, acquisition of medical equipments, and addition of hospital beds. Providers with excess capacity seldom relinquish their CONs to capacity-constrained providers out of concern of regional competition. This phenomenon of maldistributed health care resources adversely affects individuals' access to care and undermines one of the purposes of CON regulation, cost efficiency. On the other hand, in states where there is no CON regulation, capital investment by the capacity-constrained providers will eventually add health care costs to the society, while others in the same region have excess, idle capacity. In either situation, with or without CON regulation, outsourcing can represent a sound strategy for both health care providers and policy makers. Outsourcing allows capacity-constrained providers to avoid expensive expansion and still improve their functional capacity. In addition, providers with excess capacity can use outsourcing arrangements to increase their care volume and therefore improve financial performance. Through clinical outsourcing, society as a whole is spared the cost of creating duplicate resources, and access to care is improved.

Outsourcing in business has been a common practice for years. As a matter of fact, outsourcing clinical services in the health care industry already occurs implicitly and explicitly (Meyers, 2004; Romano, 2004). Outsourcing decisions might be made based on judgment, but not necessarily made with a more systemic, prospective strategic thinking (Billi et al., 2004), or with a comprehensive understanding of associated risks and benefits. Understanding environmental uncertainty is critical for any major decision making in health care. Though there are media coverage and anecdotal reports of clinical and non-clinical outsourcing in health care, leaders have not had a comprehensive discussion specifying decision criteria and uncertainty in clinical outsourcing. We perceive two elements contributing to uncertainty in clinical outsourcing: the balance of outsourcing risks and benefits, and partner alignment. Risks and benefits in outsourcing directly affect the willingness of contract parties to engage in a win-win partnership. Availability of qualified partners also contributes to the ultimate success in outsourcing. Drawing on literature from health care and other industries and experiences at our tertiary health center, we examine the risks and benefits in clinical outsourcing from multiple perspectives. A better understanding of concerns from all affected parties will help establish an all-win outsourcing partnership for all affected parties. We also provide some criteria for selecting outsourcing partners to minimize the risks in clinical outsourcing.

\section{RISKS AND BENEFITS OF CLINICAL OUTSOURCING}

Outsourcing of clinical services is a contractual arrangement that transfers patients to an external health care provider through partnership management. Outsourcing 
provides good opportunities to team up with other health care providers and benefit from each other's strengths (Chyna, 2000). Both contracting parties, as well as patients and payers, benefit from the aggregate expertise. However, these benefits do not come without risks, which represent uncertainty in strategic outsourcing.

\section{Tertiary health centers}

Based on theoretical considerations (Billi et al., 2004), good candidates for tertiary health centers to outsource are less specialized clinical services that are already well provided by local competitors. The primary benefit of outsourcing low intensity clinical services is that it allows a tertiary health center to meet patient and payer expectations for timeliness of services without costly expansion of such less specialized, or strategically less valuable services. This also allows the tertiary health center to concentrate its financial resources and management on core missions and advance its distinct specialty in order to achieve its strategic goals. From a strategic perspective, an opportunity cost is avoided by releasing resources for alternative revenue generating programs (Jennings, 1997). Another potential benefit is cost saving to payers and the tertiary health center. Cost saving can be achieved if outsourcing partners have lower unit cost for routine services. Reduction or elimination of in-house investment on outsourced services also produces cost saving. More assets are available for those more highly specialized services, to fund efforts directed to design more effective and efficient care plans, and to improve quality of care, which in turn creates more satisfied customers, both providers and patients. Another benefit is the opportunity for the tertiary health center to learn best practices from its outsourcing partners, for example in scheduling or patient communication. The tertiary health center is also more likely to receive more appropriate and specialized referrals from outsourcing partners, since each partner better understands the others' unique capabilities.

One downside of any contracting arrangement is the increased dependence and loss of control (Quinn and Hilmer, 1994; Renner and Palmer, 1999). In an outsourcing arrangement, the tertiary health center depends on its contractors to provide care with high quality and appropriateness. Without the right contract, understanding, and partner selection, the tertiary health center will have little influence over the partner's clinical practice, including appropriateness, quality, and efficiency. Negotiations, backed up in contracts, must include agreement to practice according to common evidence-based guidelines with commitment to measurement and improvement. Since seamless communication is essential in quality patient care (Renner and Palmer, 1999; Procop et al., 2003), the tertiary health center may establish a web-based integrated clinical information system (CIS) that facilitates coordination among its clinicians. Outsourcing partners would only have access to this system if the technologic, logistic, and legal barriers to CIS sharing are overcome. Without such sharing, the flow of clinical information will likely be disturbed and care coordination may be compromised.

Moreover, the ability and flexibility of the tertiary health center could be undermined in responding to environmental change, since it would not be prudent for the tertiary health center to make important strategic decisions without input from its 
outsourcing partners. A loss of strategic flexibility should be one of main strategic concerns (Quinn and Hilmer, 1994). The tertiary health center may even risk losing some of its patient base if its outsourcing partners are not willing to (or contractually bound to) refer patients back to the tertiary health centers for other non-outsourced services or higher level of medical care. An example would be the high-risk obstetrics and neonatology that arise in obstetrics patients referred to a community provider under an outsourcing agreement. Staff members at the outsourcing partners, many trained by the tertiary health center to do the complex procedures, may want to acquire the equipment and resources to perform high-end complex procedures, and avoid the referral back to the tertiary health center. It is also possible that patients will be so satisfied with the care provided by the outsourcing providers that they decide to switch more of their health care to these providers. In an extreme scenario, the tertiary health center might lose the critical skill or the residual capacity to provide the clinical services when the tertiary health center decides to retract from the outsourcing contract. It could be difficult to terminate the outsourcing contract if the tertiary health center becomes so dependent on the outsourcing partners, or the cost associated with switching to other partners is unacceptably high (Jennings, 1997).

One barrier the tertiary health center may face is the challenge from payers, who contract with the tertiary health center, not a subcontractor (outsourcing partner), to provide the services based on the tertiary health center's reputation. Other barrier includes the tertiary health center's own physicians who may perceive the outsourcing partners as competitors. Outsourcing clinical services in a more complete form denotes no investment in growth, downsizing, or even eliminating certain clinical units. This radical measure could solicit backlash from tertiary health center's employees being affected, and could lower morale and produce uncertainty among employees. If the tertiary health center holds out the partner as a high quality provider (voucher for them), the tertiary health center may bear some legal liability and certainly some public relation liability if quality is poor.

\section{Outsourcing partners}

For the health care partners that hold outsourcing contracts with a tertiary health center, the first foreseeable benefit is increased patient volume and revenues. They can also benefit from the clinical guidelines shared by the tertiary health center to improve quality of patient care. The initial outsourcing arrangement for certain clinical service may open the doors for future collaboration and sharing, even not in the formal format of legal contract. Through collaboration, partners would enjoy the expanded knowledge and access to the cutting-edge practice. They may enjoy the reputation and marketing effect associated with the tertiary health center. However, such sharing of best practices often is difficult due to conflicts in organizational culture (Chyna, 2000) and perceived threats to autonomy, with quality and performance being monitored by a tertiary health center. Adopting or adapting to a new practice protocol may result in initial interruption in clinical practice, with added cost associated with the adjustment. Disruption of patient flow is foreseeable when patients return to the tertiary health center for their regular care. 


\section{Patients (receiving outsourced services)}

As the ultimate care recipients, patients also enjoy benefits and face risks in outsourcing arrangement. For patients with tertiary health centers that are over their volume capacity, they usually wait a long time before an appointment can be scheduled. With an outsourcing arrangement, patients are referred to health care providers that have capacity for prompt services and are endorsed by tertiary health centers. However, patients might feel betrayed and abandoned simply because they are not able to receive care from providers affiliated with the tertiary health center they have long trusted and selected for their episode of care. It is likely for patients to experience either geographic convenience or inconvenience, depending on the relative locations of their home or work, tertiary health centers, and outsourcing providers. The conditions that outsourcing partners follow the practice protocol established by tertiary health centers with quality monitoring as top priority are intended to ensure patients that quality of care would not slack. Nevertheless, these assurance mechanisms do not necessarily prevent patients from suspecting reduced quality of care or less specialized expertise that they might be receiving. Further, if the information sharing and referral process between a tertiary health center and outsourcing partners do not flow smoothly as expected, the coordination and continuity of care suffers, and ultimately patients suffer more in an already complex, hierarchical, and inefficient health care delivery system.

\section{Payers and physicians requesting services from tertiary health center}

The most obvious gain from outsourcing arrangements for payers and physicians who request services from the tertiary health center is better access needed by their covered population to both services outsourced and high complexity services not outsourced. Payers can also realize lower cost if the outsourcing providers have lower unit cost. Meanwhile, payers and requesting physicians may as well concern about lower appropriateness of care provided by outsourcing providers and patient complaints due to confusion in outsourcing arrangement or perception of reduced quality of care.

\section{CRITERIA FOR PARTNERS}

A careful selection of outsourcing partners is essential to ensure a sustained collaborative relationship among health care providers and minimize the risks involved in an outsourcing contract (Allen and Chandrashekar, 2000). A major concern for a tertiary health center in strategic outsourcing is resource dependence and loss of control. An intuitive solution is for the tertiary health center to maintain capability by still offering outsourced services internally, though probably in a reduced scale, rather than completely outsourcing the services to external partners. Some non-health care companies have taken this approach to preserve their internal expertise (Allen and Chandrashekar, 2000). Alternatively, it would be desirable to contract with more than one partner for the same outsourced service to diversify the 
Table 1. Criteria in selecting outsourcing partners

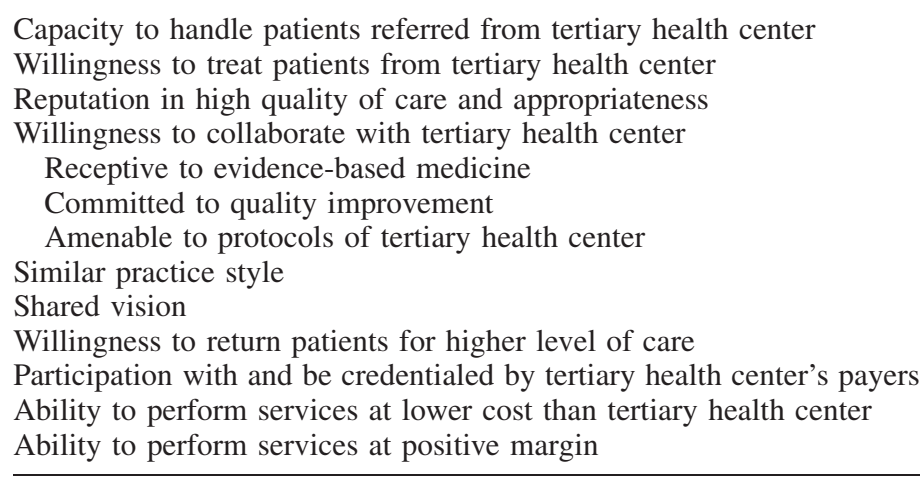

risk of dependence upon any of them, especially when there are numerous qualified candidates. The existence of alternate partners would increase a tertiary health center's autonomy by decreasing its dependence on only one partner (Cook, 1977).

A set of criteria in considering potential outsourcing partners is listed in Table 1. An obvious criterion for potential partners is that they not only have capacity but also are willing to provide care to patients referred from a tertiary health center. Patient safety and quality of care are among concerns in outsourcing clinical services (Procop et al., 2003; Chin, 2004). To ensure quality of care patients will be receiving from outsourcing providers, partners should have reputation in providing good quality of care, high appropriateness, and a commitment to continuous quality improvement. The potential partners should also desire to establish a long-term, collaborative relationship with the tertiary health center for strategic or marketing goals. Quality of care may suffer because of less commitment in an unstable, shortterm partnership (Meyers, 2004). Collaboration means both parties perceive each other more as partners and less as competitors, working together for the sake of patients' well being and for mutual benefit. As one top priority, the partners must be willing to adopt evidence-based practice guidelines, or comply with treatment protocols developed or formalize by the tertiary health center. Ideally they would be willing to participate in joint quality monitoring with the tertiary health center. In addition to developing accountability, joint monitoring programs also works to strengthen the partnerships (Lanser, 2000). A shortcut to such successful partnership is to consider those with practice styles and vision similar to those of tertiary health center providers. Otherwise, connecting the cultural difference will be one critical management practice for successful outsourcing (Roberts, 2001).

A practical concern arises when patients require higher level of care. A good candidate for outsourcing will be those who will return patients to the tertiary health center for more complicated services. Unless compelling reasons not to do so, a legal aspect of outsourcing prefers selecting partners who participate with and receive credentials from payers such as managed care organizations, so that the managed care organizations are able to fulfill their accreditation requirements by the National Committee for Quality Assurance (NCQA). For the benefit of cost saving for both the 
tertiary health center and payers, outsourcing partners should be able to provide services at lower cost than the tertiary health center (Egger, 1999). Operation at positive margin by providing outsourced services to the tertiary health center patients would definitely appeal to potential partners. A win-win situation is essential to a successful, sustainable partnership.

\section{CONCLUSION}

Outsourcing clinical services represent core structure changes for a tertiary health center, and are seldom an easy, simple step to take. Outsourcing clinical services deserve strategic thinking of missions and competitive strength a health care provider embraces to distinct itself from its competitors. In this article, we discuss clinical outsourcing mainly from the perspective of tertiary care providers, though most of the considerations are applicable to less specialized health care providers. For example, cautions on referral practices back to the tertiary care center are also relevant to secondary hospitals. Depending on the mission of an organization and its goal for clinical outsourcing, outsourcing benefits and risks as well as criteria for partner selection may receive differential weights in decision making. Mackey et al. (2004) summarize clinical outsourcing as 'using the right resources for the right reasons.' To control costs and enhance efficiency, community health centers may prefer outsourcing partners that can perform services at lower costs. On the other hand, clinical outsourcing allows a tertiary health center to fulfill its core missions while still enabling it to stay committed to its community services and quality of care. Thus, reputation in quality of care provided by partners will definitely receive more preference. In addition, the risk of losing patient base and the worry about coordination and continuity of care need to be considered along with concerns from other parties affected by outsourcing decisions. A good partner selection and management will reduce outsourcing risks, leading to an all-win situation.

\section{REFERENCES}

Allen S, Chandrashekar A. 2000. Outsourcing services: the contract is just the beginning. Bus Horiz 43(2): 25-34.

Bazzoli GJ, Brewster LR, Liu G, Kuo S. 2003. Does U.S. hospital capacity need to be expanded? Health Aff (Millwood) 22(6): 40-54.

Billi JE, Pai CW, Spahlinger DA. 2004. Strategic outsourcing of clinical services: a model for volume-stressed academic medical centers. Health Care Manage Rev 29(4): 291-297.

Center for Financing, Access, and Cost Trends. 2004. Medical Expenditure Panel Survey Household Component, 2004. Agency for Healthcare Research and Quality: Rockville, MD.

Chin T. 2004. Radiologists weigh in on outsourcing of imaging work. Am Med News 47(22): 23.

Chyna JT. 2000. From alliances to outsourcing: making good connections. Health Exec 15(3): $12-16$.

Cook KS. 1977. Exchange and power in networks of interorganizational relations. In Organizational Analysis: Critique and Innovation, Benson JK (ed.). Sage Publications Inc: Beverly Hills. 
Egger E. 1999. Possible solutions to the bottom line blues. Health Care Strateg Manage 17(5): $19-20$.

Green LV. 2002. How many hospital beds? Inquiry 39(4): 400-412.

Greenblatt J. 2002. Access to Urgent Medical Care: 2001. Statistical Brief No. 8. Agency for Healthcare Research and Quality: Rockville, MD.

Institute of Medicine. 2001. Crossing the Quality Chasm: A New Health System for the 21st Century. National Academy Press: Washington, DC.

Jennings D. 1997. Strategic guidelines for outsourcing decisions. Strategic Change 6(2): 8596.

Lanser EG. 2000. The ins and outs of outsourcing. Health Exec 15(2): 58-59.

Mackey TA, McNiel NO, Klingensmith K. 2004. Outsourcing issues for nurse practitioner practices. Nurs Econ 22(1): 21-26.

Meyers S. 2004. ED outsourcing: is it good for patient care? Trustee 57(4): 12-14, 19-20.

Procop GW, Winn W, Microbiology Resource Committee, College of American Pathologists. 2003. Outsourcing microbiology and offsite laboratories. Implications on patient care, cost savings, and graduate medical education. Arch Pathol Lab Med 127(5): 623-624.

Quinn JB, Hilmer FG. 1994. Strategic outsourcing. Sloan Manage Rev 35(4): 43-55.

Renner C, Palmer E. 1999. Outsourcing to increase service capacity in a New Zealand hospital. J Manag Med 13(4-5): 325-338.

Roberts V. 2001. Managing strategic outsourcing in the healthcare industry. J Healthc Manag 46(4): 239-249.

Romano M. 2004. Outsourcing everything. Accelerating use of outsourcing adds to public policy debate while raising questions about hospitals' 'charitable' status. Mod Healthc 34(14): 24-26, 34.

Strunk BC, Cunningham PJ. 2001. Treading water: Americans' access to needed medical care, 1997-2001. Tracking Report March(1): 1-6. 